\title{
Study on the Thought of "Frugality" in Japanese Product Design
}

\author{
Junying Shi
}

\author{
School of Fine Arts and Design, Hubei College of Engineering, Xiaogan, Hubei 432000 \\ shijunying1981@126.com
}

Keywords: Japanese style; Product design; Frugality

\begin{abstract}
The morphology exhibited by Japanese design products has a certain degree of uniformity to some extent. the unity of this design form was defined as Japanese style in the design community. The design morphology of Japan is largely influenced by the Japanese tradition of "frugality", which runs through ancient times to modern, manifested in all aspects of the Japanese's life. When the European modern design pioneers found that the Japanese style with the elements they are seeking, the Japanese design style began to integrate with the world and be recognized by the design community.
\end{abstract}

\section{Overview of Japanese Product Design Features}

Thought Characteristics of Japanese Product Design. Japan's original design always be with a unique national temperament, this temperament not only was derived from the simple reproduction of traditional national style, but also is the "redesign" arising from the national philosophy of creation combined with global design trends. From the international perspective, the Japanese designs are full of modern sense; but from the traditional point of view, the local properties of Japanese designs are also very strong. The balance of this state pushed the design onto the international arena.

Japan design with an eye to design concept of "designs come from life, and applied in life", and strive to avoid the split phenomenon between material needs and cultural needs in the industrial environment, in the Japanese original design, they show that the people pursuit the nature of culture, memory and emotion, and thus create a sense of identity in the audience's emotions naturally, emotional resonance generated by this sense of identity form a unique aesthetic experience. The use of blank can often be found in Japanese design works, this form reflects its understanding of "virtual" . This very kind of "nothingness" feeling can reflect the mood of all things inclusive, and then this kind of "nothingness" thought can further trace to "man feel peaceful and lonely, nothingness, it is this world peace and moral nature" mentioned by Zhuangzi in the "Zhuangzi, extension articles, deliberately, the fifteenth".

Takumiru fog clock were designed by Japan idea design company, although the designer respects the concept of nothingness, but the second hand of the watch are ticking all the time, reminding the passage of time, this strong contrast makes this product more unique design sense.

Morphology Characteristics of Japanese Product Design. Today, when mentioned the Japanese design, people always sum up and describe them habitually as "small", "fine", "functional diversity", as if these words have become a text forward of Japanese design style. London's TKO design creative director Andrew Davey used a large number of Japanese modern design case to discuss the six characteristics of the Japanese industrial product design, namely, ideal, the pursuit of perfection, emphasizing the combination of new technology and beauty, applying the principle of minimization, closely following the consumer and continuous innovation in the form and function. We are not unfamiliar with the six-point summary of the Japanese modern design summarized by Andrew David, because the widespread spread and application of Japanese design products on a global scale has produced enough influence to make us form sense impression. to some extent, Japanese modern design just confirms the sentence of " things with local colors will be easily integrated into the world" in the letter of Mr. Lu Xun writing to Chen Yancai .

Taking the common Japanese product MUJI without brand for example, their advocating the natural, simple, simple way of life have been greatly accepted by the people. Its products removed 
the brand, eliminating the need for unnecessary design, removing all unnecessary processing and color, simple just the material and the function itself. In the development of commodity, MUJI have developed strict rules on the design, raw materials, prices. For example, clothing category need to strictly abide by design principles of no pattern, checkered, stripes and others, only black and white, brown are used, no matter how popular of the year colors, they never exceed the design principles to develop goods.

The International Influence of Japanese Product Design. Japanese design has clearly formed its own unique style features, until today the world's design theorists are continually taking the Japanese design to the topic or as the object of study. Japanese design style is gradually found in the commercial competition of global market for Toyota, Sony, Canon and a series of Japanese brands. Further analysis, it will find the real decisive role is not those designs themselves, but ideas hidden in the subsequent creation.

The case of Honda researching the robot made in ASIMO and SONY company to develop AIBO is well-known globally. In the civilian product design, the birth of such as "ripple cup" shoes and 645 and 645N II type camera produced by Fuji Bin Optics Co., Ltd.

Honda 125ccRC143 motorcycle adhering to the characteristics of Honda's high-tech aesthetics, relying on he perfect combination of the beautiful appearance and the high-speed rotation of the four-stroke dual camshaft engine, achieving Tom Phillips Spanish Grand Prix laurel, it not only has become one of the criteria for 60's Japan "excellent design award" in last century, is also reflected in its product design until today. PLAY STATION game launched by SONY is the most typical representative, relying on its rich software resources and ergonomic product design fully embodying the man-machine engineering, PS has become an immortal brand, standing in the minds of countless consumers. Fuji's single-use camera brings innovation to the camera industry, and since then, the camera did not belong to the expensive consumer level, it can be "one-time thing" cheap products. This example is common in Japanese product design cases, and the design concept of these products led to the development of the global industrial capital, and inspiring the endless creativity of the global designer.

\section{History and Current Situation of Frugality in Japanese Products}

The Frugal Design Concept in the Japanese Traditional Instruments. Before the establishment of the Shimen mind, the handicraftsman might not have considered the ethical rules of "frugality" to carry out creation, but when in the design of equipment, they can integrate into the "frugality" of the design concept by obeying the historical and natural conditions. Japanese-style traditional folding fan held a very clear "frugal" color through the analysis of design[10]. The "frugal" performance of Folding fan rooted in the effective use and saving of space. The purpose of People using the fan is to cool the air, but carrying a fan will add a certain amount of space to the normal person. Folders clearly provide a more reasonable solution, which provides a relatively stable balance between function and space.

The air in Japan is humid and climate changing, people invented the material called stacking to lay the floor. The stacking is often translated into "tatami" by Japanese, and it is made by weaving and bundles the straw, so it can play a good moisturizing effect. People can sleep on the tatami directly, and when they get up, people can fold the bedding into the closet, and take out the table, then the bedroom immediately is turned into a restaurant. And from the invention of tatami, the consideration on the use of space scales for Japanese is very mature.

Small to folding fan, large to traditional residential buildings, this problems are all around how to use the space resources reasonably and effectively. They are reflected the point of view of the maximum to curb the waste and take full advantage of things that the Shitian Meiyan emphasized. This once again proved that Shitian's "frugality" ethic concept has the meaning in ancient times and modern, it is not only the summary of advanced culture of ancestor, but also the theoretical basis of the future of Japan' s modern design form.

The Frugality Concept in the Japanese Modern Design. In my opinion, no product can be more representative than the Walkman to reflect Japanese "frugality" design concept. In this only 
palm-sized rectangular box, it contains infinite charm, which itself inherited the Japanese traditional ideas, while laid the foundation of the prototype for the future of the portable music player products. It completely changed the traditional way of listening for human beings, but also promote the Japanese culture to the world stage by taking this opportunity. I learned: the chairman of Sony's Akio Morita suddenly came the concept into being one day, under the insistence, through the collective labort of he Sony R \& D team. The so-called business leaders, its essence is actually businessmen. Ishida's philosophy not only make the merchant class pursue the frugality, but also created the consumer groups with a consensus. Therefore, the success of the Japanese design first came from the identity of the nation.

\section{The Form of Frugality}

The Miniaturization of The Design. The "frugality " of folding fan and other traditional designs are mainly reflected in the rational allocation of space resources, Walkman design also reflects this point. Before the appearance of the Walkman, people generally listen to music through a larger tape recorder. This kind of tape recorder is very inconvenient to carry, because its space occupation is larger. While the volume of the Walkman can accommodate a cartridge, which saves the cost of space to the limit. This way of listening is disruptive, and it even changes the face of today's society.

Walkman design is forward-looking, it is the first time to build an economic and reasonable mode of work between people and portable multimedia devices. And with the progress of science and technology, this model is gradually copied by more and more products. Walkman's most clear frugality embodied in the miniaturization of the form, this form of expression in the Japanese design is very common. These designs reflect the same "frugality" ethic. Unity of design concept was shown through a variety of products, the resultant force must create a distinctive image of the Japanese design style.

The Philosophy of Concentration within the Product. Concentration of the product generally refers to the concentration of the functions, because the purpose of people usually to buy products is not the product itself, but rather its use functions. If a product can be a variety of functions, it shows that it maximizes to use the space resources.

Play station portable handheld gamer released by the Sony company in December 2004. Same with the pursuit of maximizing the function of the phone, the Japanese traditional "frugality" consciousness once again put concentrated design concept into the PSP design and development. Consumers simply buying the required functional modules can make it become a new product their purchased ago, this consumer concept is clearly welcomed by consumers, and it also fully reflects the "frugality" consciousness of the economic rationalization.

The Principle of Conservation Design. The universality of the "frugality" ethic in Japanese society makes the design concept of saving embody in the Japanese design commonly. For the large number of consumer electronic products in the daily life, the object of its savings is the energy source clearly.

Panasonic RQ-SX72 Walkman can achieve to play 72 hours continually through a plug-in AA batteries with built-in chewing gum battery. Sony's digital Walkman NW-S202F, its built-in lithium battery can achieve continuous playback of 3 hours after being charged 3 minutes. The battery in fashion watch G2900 that Japan Casio company launched with the same price can continue to use nearly 10 years. Today, Casio has begun to promote solar watch in the market, this watch has no battery, but the use of advanced solar drive system to supplement the energy. The advantage of this system is that not only sunlight can be converted into electrical energy, even weak fluorescent lamps can be its power resource.

\section{The Influence of "frugality" on Product Design and Its Enlightenment}

Miniaturization design, concentration design, economical design... The composition of these forms constitutes a distinctive impression of national design style. In terms of design, its success depends largely on market performance evaluation. But the design excellence is not limited to the real 
economic benefits it brings, more valuable is that it can promote national culture and traditional spirit through a variety of design languages to the world and won the recognition.

All things are designed today, a nation-specific design style is particularly important. To create a national style must require us to return to review the development process of the nation. Natural factors created the Japanese "frugality" consciousness, these consciousness was only in the subconscious by the design of Japanese traditional instruments to reflect. The social universality of Shimen psychology, the various sectors of agriculture, industry and commerce have formed the pursuit of "integrity" and "frugality", which has become an invisible force that restrains Japanese society.

Therefore, the various class as the buyer and the merchant as the seller have greatly consistency in the ideology, so the Japanese design in the country is very easy to get approval. And when the modernist system was established, the Japanese design was again recognized by the world. This is because when the European modern design pioneers were struggling to explore the best form of modern design, they found that the Japanese design provides a very useful example.

\section{References}

[1] H.L. Lan. Zen thought in the application of product design [J]. Journal of Zhengzhou University of Light Industry: Social Science Edition, 2013

[2] Y. Li. Analysis on modern glass design development of Scandinavian [J]. Popular Literature: Academic Version, 2013

[3] D.D. Lu. Discussion on the importance of detail awareness in product design [J]. Popular Literature, 2014 